\title{
Application of Cellulose and Cellulose Derivatives in Pharmaceutical Industries
}

\author{
Javad Shokri and Khosro Adibkia
}

Additional information is available at the end of the chapter

http://dx.doi.org/10.5772/55178

\section{Introduction}

Cellulose probably is the most abundant organic compound in the world which mostly produced by plants. It is the most structural component in herbal cells and tissues. Cellulose is a natural long chain polymer that plays an important role in human food cycle indirectly. This polymer has versatile uses in many industries such as veterinary foods, wood and paper, fibers and clothes, cosmetic and pharmaceutical industries as excipient. Cellulose has very semi-synthetic derivatives which is extensively used in pharmaceutical and cosmetic industries. Cellulose ethers and cellulose esters are two main groups of cellulose derivatives with different physicochemical and mechanical properties. These polymers are broadly used in the formulation of dosage forms and healthcare products. These compounds are playing important roles in different types of pharmaceuticals such as extended and delayed release coated dosage forms, extended and controlled release matrices, osmotic drug delivery systems, bioadhesives and mucoadhesives, compression tablets as compressibility enhancers, liquid dosage forms as thickening agents and stabilizers, granules and tablets as binders, semisolid preparations as gelling agents and many other applications. These polymeric materials have also been used as filler, taste masker, free-flowing agents and pressure sensitive adhesives in transdermal patches. Nowadays cellulose and cellulose based polymers have gained agreat popularity in pharmaceutical industries and become more and more important in this field owing to production of the new derivatives and finding new applications for existed compounds by pharmaceutical researchers.

\section{Classification of cellulose-based polymers}

\subsection{Cellulose}

Pure cellulose is available in different forms in the market with very different mechanical and pharmaceutical properties. The difference between various forms of cellulose is related 
to the shape, size and degree of crystallinity of their particles (fibrous or agglomerated). Microcrystalline cellulose (MCC) is the most known cellulose which extensively used in pharmaceutical industries. MCC grades are multifunctional pharmaceutical excipients which can be used as compressibility enhancer, binder in wet and dry granulation processes, thickener and viscosity builder in liquid dosage forms and free-flowing agents in solid dosage forms. Mechanical properties of MCC grades are greatly influenced by their particle size and degree of crystallization. In recent years the new grades of MCC are prepared with improved pharmaceutical characteristics such as silisified MCC (SMCC) and second generation MCC grades or MCC type II (MCC-II). These grades are prepared by coprocessing of cellulose with other substances such as colloidal silicon dioxide or by special chemical procedures. Other types of available pure cellulose are powdered cellulose (PC) and low crystallinity powdered cellulose (LCPC).

Regenerated cellulose is one of the other forms of processed cellulose which produced by chemical processing on natural cellulose. In the first step, cellulose dissolves in alkali and carbon disulfide to make a solution called "viscose". Viscose reconverted to cellulose by passing through a bath of dilute sulfuric acid and sodium sulphate. Reconverted cellulose passed through several more baths for sulfur removing, bleaching and adding a plasticizer (glycerin) to form a transparent film called cellophane. Cellophane has several applications in pharmaceutical packaging due to its suitable characteristics such as good compatibility, durability, transparency and elasticity.

\subsection{Cellulose ether derivatives}

Cellulose ethers are high molecular weight compounds produced by replacing the hydrogen atoms of hydroxyl groups in the anhydroglucose units of cellulose with alkyl or substituted alkyl groups. The commercially important properties of cellulose ethers are determined by their molecular weights, chemical structure and distribution of the substituent groups, degree of substitution and molar substitution (where applicable). These properties generally include solubility, viscosity in solution, surface activity, thermoplastic film characteristics and stability against biodegradation, heat, hydrolysis and oxidation. Viscosity of cellulose ether solutions is directly related with their molecular weights. Examples of mostly used cellulose ethers are: Methyl cellulose (MC), Ethyl cellulose (EC), Hydroxyethyl cellulose (HEC), Hydroxypropyl cellulose (HPC), hydroxypropylmethyl cellulose (HPMC), carboxymethyl cellulose (CMC) and sodium carboxymethyl cellulose (NaCMC).

\subsection{Cellulose ester derivatives}

Cellulose esters are generally water insoluble polymers with good film forming characteristics. Cellulose esters are widely used in pharmaceutical controlled release preparations such as osmotic and enteric coated drug delivery systems. These polymers are often used with cellulose ethers concurrently for preparation of micro-porous delivery membranes. Cellulose esters categorized in organic and inorganic groups. Organic cellulose esters are more important in pharmaceutical industries. Various types of organic cellulose 
esters have been used in commercial products or in pharmaceutical investigations such as cellulose acetate (CA), cellulose acetate phthalate (CAP), Cellulose acetate butyrate (CAB), Cellulose acetate trimelitate (CAT), hydroxupropylmethyl cellulose phthalate (HPMCP) and so on (Heinämäki et al., 1994). The most available formulations in market which made by these polymers are enteric coated dosage forms which are usually produced applying acid resistant polymeric coats containing phthalate derivatives of cellulose esters especially cellulose acetate phthalate (Lecomte et al., 2003; Liu \& Williams III, 2002). Inorganic cellulose esters such as cellulose nitrate and cellulose sulphate are less important than organic cellulose esters in pharmaceutical industries. Cellulose nitrate or pyroxylin is a transparent compound with good film forming ability but rarely applied alone in pharmaceutical formulations due to its very low solubility in currently used pharmaceutical solvents as well as their very high flammability. The use of pure cellulose nitrate in drug formulations only limited to one topical anti-wart solution named collodion that made with $4 \% \mathrm{w} / \mathrm{v}$ concentration in diethyl ether/ethanol mixture as solvent. Cellulose nitrate/cellulose acetate mixture are also exploited to prepare micro-porous membrane filters used in pharmaceutical industries.

\section{Applications of cellulose and its derivatives in pharmaceutical industries}

\subsection{Application in bioadhesive and mucoadhesive drug delivery systems}

Bioadhesives and mucoadhesives are drug containing polymeric films with ability of adhering to biological membranes after combining with moisture or mucus compounds. Bioadhesives were developed in mid 1980s as a new idea in drug delivery and nowadays they have been accepted as a promising strategies to prolong the residence time and to improve specific localization of drug delivery systems on various biological membranes (Lehr, 2000; Grabovac et al., 2005; Movassaghian et al., 2011).

In compared with tablets, these dosage forms have higher patient compliance due to their small size and thickness. Other advantage of these drug delivery systems is their potential to prolong residence time at the site of drug absorption and thus they can reduce the dosing frequency in controlled release drug formulations. These dosage forms can also intensify the contact of their drug contents with underlying mucosal barrier and improve the epithelial transport of drugs across mucus membranes especially in the case if poorly absorbed drugs (Ludwig, 2005; Lehr, 2000). Some special polymers can be used in these formulations with epithelial permeability modulation ability by loosening the tight intercellular junctions. Some of these polymers also can act as proteolytic enzymes inhibitor in orally used adhesive formulations of sensitive drugs (Lehr, 2000).

Bioadhesives considered as novel drug delivery systems. These dosage forms are formulated to use on the skin and mucus membranes of gastrointestinal, ear, nose, eye, rectum and vagina. The main excipients of these formulations are adhesive and film-former polymer(s). Adhesive polymers are synthetic, semi synthetic or natural macromolecules 
with capablility of attaching to skin or mucosal surfaces. Very different types of polymers have been used as bioadhesive polymers. Synthetic polymers such as acrylic derivatives, carbopols and polycarbophil, natural polymers such as carageenan, pectin, acacia and alginates and semi-synthetic polymers like chitosan and cellulose derivatives are used in bioadhesive formulations (Deshpande et al., 2009; Grabovac et al., 2005). Cellulose derivatives especially cellulose ethers are widely used in bioadhesives. There are used in various types of these formulations such as buccal, ocular, vaginal, nasal and transdermal formulations alone or with combination of other polymers. More recently used cellulose ethers in bioadhesives include nonionic cellulose ethers such as ethyl cellulose (EC), hydroxyethyl cellulose, hydoxypropyl cellulose (HPC), methyl cellulose (MC), carboxymethyl cellulose (CMC) or hydroxylpropylmethyl cellulose (HPMC) and anionic ether derivatives like sodium carboxymethyl cellulose (NaCMC). Ability of polymer to take up water from mucus and $\mathrm{pH}$ of target place are important factors determining the adhesive power of polymers. Some bioadhesive polymers such as polyacrylates show very different adhesion ability in various $\mathrm{pH}$ values thus the selection of adhesive polymer should be made based on the type of bioadhesive preparation. One advantage of cellulose ethers such as $\mathrm{NaCMC}$ and HPC is lesser dependency of adhesion time and adhesion force of them to $\mathrm{pH}$ of medium in compared with polyacrylate and thiolated bioadhesive polymers (Grabovac et al., 2005). Cellulose ethers, alone or their mixtures with other polymers, have been studied in oral (Deshpande et al., 2009; Venkatesan et al., 2006), buccal (Perioli et al 2004), ocular (Ludwig, 2005), vaginal (Karasulu et al., 2004) and transdermal (Sensoy et al., 2009) bioadhesives. In some studies, other groups of adhesive polymers or polysaccharides are used with cellulose ethers to improve their adhesion characteristics such as adhesion time and adhesion force. Concurrent use of polyvinyl pyrrolidone (PVP), hydroxypropyl beta cyclodextrin, polycarbophil, carbopol(s), pectin, dextran and mannitol with HPMC, HEC or NaCMC have been reported in the literatures. (Karavas et al., 2006).

\subsection{Application in pharmaceutical coating processes}

Solid dosage forms such as tablets, pellets, pills, beads, spherules, granules and microcapsules are often coated for different reasons such as protection of sensitive drugs from humidity, oxygen and all of inappropriate environmental conditions, protection against acidic or enzymatic degradation of drugs, odor or taste masking or making site or time specific release characteristics in pharmaceuticals to prepare various modified release drug delivery systems such as sustained release, delayed release, extended release, immediate release, pulsatile release or step-by-step release dosage forms (Barzegar jalali et al., 2007; Gafourian et al., 2007). Both ether and ester derivatives of cellulose are widely used as coating of solid pharmaceuticals. Cellulose ethers are generally hydrophil and convert to hydrogel after exposing to water. Although, some of the cellulose ethers e.g. ethyl cellulose are insoluble in water but majority of them such as methyl, hydroxypropyl and hydroxylpropylmethyl cellulose are water soluble. Both of soluble and insoluble cellulose ethers can absorb water and form a gel. After exposing of these coated dosage forms with water, the coating polymers form to hyrogel and gradually dissolve in water until disappear 
but the insoluble cellulose ether coatings remain as a viscose gel around tablets and drug release is performed by diffusion of drug molecules within this layer. These two types of dosage forms called dissolution-controlled and diffusion-controlled drug delivery systems, respectively. Despite cellulose ethers, the cellulose esters are generally water insoluble or water soluble in a distinct $\mathrm{pH}$ range. These polymers like cellulose acetate (CA), cellulose acetate phthalate (CAP) and cellulose acetate butyrate $(\mathrm{CAB})$ do not form gel in presence of water and they are widely used for preparing of $\mathrm{pH}$ sensitive and semi-permeable microporous membranes. These membranes are employed for wide variety of controlled release coating of pharmaceuticals especially in enteric or osmotic drug delivery devices. These polymers are benefited to make different cellulosic membrane filters applied in pharmaceutical industries.

\subsection{Application in extended release (ER) solid dosage forms}

\subsubsection{In coated extended release formulations}

Extended release pharmaceuticals refer to dosage forms that allow a twofold or greater reduction in frequency of the drug administration in comparison with conventional dosage forms. These formulations can be made as coated or matrix type. Coated ER formulations are generally made with water insoluble polymeric film coating with or without gel-forming ability. The dominant mechanism of drug release in coated ERs is diffusion whereas in matrix type of ERs, erosion of matrix is the main mechanism of drug release. The most used cellulosic polymer in these modified release dosage forms is ethyl cellulose. Ethyl cellulose is completely insoluble in water, glycerin and propylene glycol and soluble in some organic solvents such as ethanol, methanol, toluene, chloroform and methyl acetate. Aqueous dispersions of ethyl cellulose such as Surelease ${ }^{\circledR}$ (Colorcon) or Aquacoat ${ }^{\circledR}$ (FMC BioPolymer) or its organic solutions can be used for coating of extended release formulations. After ingestion of these formulations, an insoluble viscose gel is forming around the tablet which doesn't allow to drug to freely release from dosage form. Drug molecules should pass across this barrier by diffusion mechanism to enter the bulk dissolution medium and thusthe release duration is extended much more than the same uncoated conventional formulation. Larger solid pharmaceuticals like tablets can be coated with rotating pan coaters whereas the smaller types as pills, beads or granules are coated with fluidized bed or air-suspension coater equipments. Because of water insolubility of EC, it is often used in conjunction with water soluble polymers such as MC and HPMC in aqueous coating liquids (Frohoff-Hülsmann et al., 1999a, 1999b). EC solutions in organic solvents such as ethanol can be thickened by HPMC or HPC (Rowe, 1986; Larsson et al., 2010). Water soluble cellulosic polymers with higher amounts can be used as pore former in micro-porous types of extended release and enteric systems. Using of plasticizers is necessary for achieving acceptable coating of pharmaceuticals by these polymers. EC is compatible with commonly used plasticizers such as dibutyl phthalate, diethyl phthalate, dicyclohexyl phthalate, butyl phthalyl butyl glycolate, benzyl phthalate, butyl stearate and castor oil. Other plasticizers such as triacetin, cholecalciferol and $\alpha$-tocopherol also have 
been used in EC film coats (Arwidson et al., 1990; Kangarlou et al., 2008). The molecular weights of ECs are in a wide range and different grades of them are existed from 4 to 350 (Colorcon official website). Concentration of $5 \% \mathrm{w} / \mathrm{v}$ from these EC grades in toluene/ethanol mixture at $25^{\circ} \mathrm{C}$ can produce about 3 to $380 \mathrm{cp}$ viscosity.

\subsubsection{In extended release polymeric matrices}

Matrices are very simple and efficient systems for controlling drug release from dosage forms. Production of these systems is less time consuming and no needs to special or sophisticated equipments. Majority of ER matrices are made by a simple mixing of drug, polymer(s) and filler followed by one or two stage compaction process. Polymeric matrices as drug delivery systems are very important in developing of modified release dosage forms. In these devices, the drug is dispersed either molecularly or in particulate form within a polymeric network. The main types of drug delivery matrices included swellable and hydrophilic monolithic, erosion controlled and non-erodible matrices (Roy et al., 2002). The use of hydrophilic matrices has become extremely popular in controlling the release rate of drugs from solid dosage forms due to their attractiveness in the case of economic and process development points of view (Conti et al., 2007). During the last two decades, hydrophilic swellable polymers have been widely used for preparation of controlled release matrix tablet formulations. Although various types of rate controlling polymers have been used in hydrophilic matrices, cellulose derivatives especially cellulose ethers are probably the most frequently encountered in pharmaceutical literatures and the most popular polymers in formulation of commercially available oral controlled release matrices. They good compressibility characteristics so they are easily converted to matrices by direct compression technique. In contact with an aqueous liquid, i.e., dissolution medium or gastrointestinal fluid, the hydrophilic polymers present in the matrix swell and a viscose gelatinous layer formed in outer surface of matrix. This layer controls the drug release from matrix. Drug molecules can release out of system by diffusion across this layer. Viscosity of the gel layer is a critical rate-controlling factor in drug release rate from matrices. Erosion of polymeric matrices also can influence the release of the drug from system. Increasing viscosity of the gel, gives rise to increase the resistance against polymer erosion and drug diffusion resulting in reduction of the drug release rate. Various types of cellulose derivatives have been used in formulation of hydrophilic polymeric matrices such as HPMC, NaCMC, CMC, HEC, HPC and EC with different molecular weights (Barzegar-jalali et al., 2010; Javadzadeh et al., 2010; adibkia et al., 2011; Asnaashari et al., 2011). Both of soluble and insoluble cellulose ethers can be used in hydrophilic polymeric matrices due to their hydrophilic nature and ability of them to forming gel in aqueous media. The highest swelling power and hydration rate among cellulose ethers is related to HEC (Saša et al., 2006) but the mostly used cellulose ether is hydrophilic matrices is HPMC due to its excellent swelling properties, good compressibility and fast hydration in contact with water (Ferrero et al., 2008, 2010; Nerurcar et al., 2005). For achieving the good release characteristics, mixtures of various cellulose ethers or mixtures of different grades of a distinct polymer with different ratios can be used based on the intended release rate of 
controlled release system (Chopra et al., 2007). Some specialized hydrophilic matrices can be made with cellulose ethers for special purposes for example, HPMC matrices with alkalizing buffers like sodium citrate for protection of acid labile drugs have been investigated (Pygall et al., 2009).

\subsection{Application in osmotic drug delivery systems}

In recent years, considerable attention has been focused on development of novel drug delivery systems (NDDS). Among various NDDS available in the market, oral controlled release (CR) systems hold the major market share because of their advantages over others. Majority of oral CR systems fall in the category of matrices, reservoirs and osmotic devices. Among various types of CR systems, osmotic devices are considered as novel CR systems (J. Shokri et al., 2008a). These formulations utilize osmotic pressure as energy source and driving force for delivery of drugs. Some physiological factors such as $\mathrm{pH}$, presence of food and gastrointestinal motility may affect drug release from conventional CR systems (matrices and reservoirs), whereas, drug release from oral osmotic systems is independent of these factors to a large extent. A classic osmotic device basically consists of an osmotically active core surrounded by a semi-permeable membrane (SPM) and a small orifice drilled through SPM using LASER or mechanical drills. In fact, this system is really a coated tablet with an aperture which acts as drug delivery port (figure 1). This type of devices is called

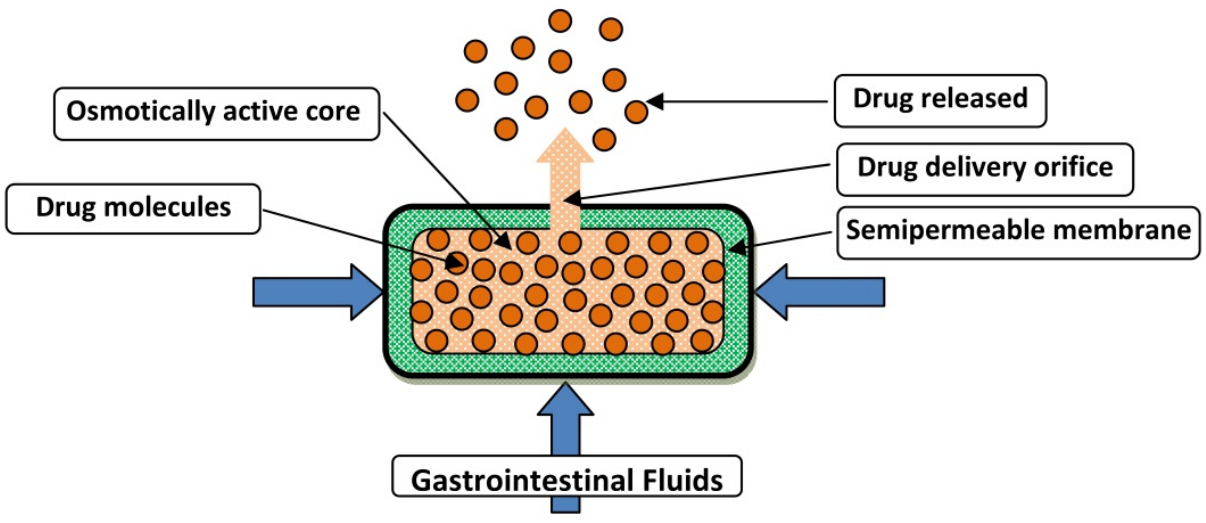

Figure 1. Schematic diagram of an EOP osmotic system.

monolithic or elementary osmotic pumps (EOPs). The more sophisticated osmotic devices have bi-layer (push-pull systems) or tri-layer (sandwich osmotic pumps) cores consisted of an osmotically active drug layer and polymeric layer(s) in one or two sides. Some of osmotic systems called asymmetric membrane or controlled porosity osmotic pumps have not any orifice in their SPM (wang et al., 2005). In these devices, water soluble polymers are used in their SPM as pore formers. Pore formers dissolve after exposing of dosage form to aqueous media and numerous micro pores are created in SPM for drug delivery reason. When an osmotic tablet exposed to an aqueous environment, water pumps from outside into the 
system due to the great osmotic pressure difference between two sides of SPM. Pumping of water into the system increases the inner hydrostatic pressure leading the saturated drug solution to flow through the small drug delivery orifice or micro pores (in the case of asymmetric membrane devices). Because of high difference of osmotic pressure between two sides of SPM, the osmotic pressure gradient remain constant and thus, the release rate of drug from these devices is almost constant and independent to environmental conditions. EOPs are the most commercially important osmotic devices so that more than 240 patents have been devoted. Procardia $\mathrm{XL}^{\circledR}$ and Adalat CR (nifedipine), Acutrium ${ }^{\circledR}$ (phenylpropanolamine), Minipress $\mathrm{XL}^{\circledR}$ (prazocine) and Volmax ${ }^{\circledR}$ (salbutamol) are examples of EOPs available in the market (J. Shokri et al., 2008a; Nokhodchi et al., 2008).

\subsubsection{In SPM formulation of osmotic systems}

As noted earlier, each osmotic delivery system is consisted of two main components included osmotically active core and semi-permeable membrane (SPM). Cellulose acetate (CA) is the mostly used polymer in formulation of SPM in all types of osmotic drug delivery devices. This polymer is the most important cellulose ester derivative with good film forming ability and mechanical characteristics for using in osmotic systems. CA is insoluble in water in both acidic and alkaline conditions. The CA films are only permeable to small molecules such as water while larger molecules like organic drugs can not pass through them. Plasticizers are used in SPM composition for improving the flexibility and mechanical properties of membrane. Various types of plasticizers have been used in formulation of osmotic pharmaceuticals such as castor oil, low and medium molecular weights polyethylenglycols (PEGs), sorbitol, glycerin, propylene glycol, triacetine, ethylene glycol monoacetate, diethyl phthalate, diethyl tartrate and trimethyl phosphate (J. Shokri et al., 2008a, 2008b; Prabakaran et al., 2004; Makhija \& Vavia, 2003; Liu et al., 2000a, 2000b; Okimoto et al., 1999). Generally, the mixture of hydrophilic and hydrophobic plasticizers is used for producing the intended drug release characteristics. In controlled porosity osmotic pumps (CPOPs), the additional components such as pore formers are needed. The most efficient pore formers are hydrophilic polymers with high water solubility properties. Water soluble cellulose ether derivatives can be used as pore former in SPM of these devices. Low molecular weight grades of these polymers are suitable for this purpose due to their faster dissolution rate and lower viscosities. Low molecular weight MCs and HPMCs have been used as pore former in CPOP formulations. Central cores are coated with a coating formulation containing SPM components such as film former (CA), pore former(s) and plasticizer(s) dissolved or dispersed in a suitable liquid base. Acetone/ethanol mixtures are generally used as solvent system to dissolve cellulose acetate in coating liquid (J. Shokri et al., 2008a; Nokhodchi et al., 2008; M.H. Shokri et al., 2011). In some studies, cellulose acetate is used as fine particles suspended in an aqueous medium for coating of osmotic cores (Liu et al., 2000b). Ethyl cellulose (EC) and ethylhydroxyl propyl cellulose also have been used as SPM of osmotic devices in some studies but permeability of these membranes is lower than CA membranes. In these formulations, hydrophilic cellulose ether derivatives such as 
HPMC have been used for improving SPM permeability (Marucci et al., 2010; Wang et al., 2005; Hjärtstam et al., 1990).

\subsubsection{In central core of osmotic systems}

Central core of an osmotic pump is generally a simple compressed tablet basically consisted of the active drug(s), osmotically active agent(s), hydrophilic polymer(s) and other commonly used ingredients such as filler, compressibility enhancer, free flowing agent and lubricant. In one compartment devices (EOPs and controlled porosity OP), these polymers mixed with other ingredients and compressed to a tablet whereas in two layered (Push-Pull systems), or tri layered (Sandwich systems) cores, these polymers compressed in one or two separated layer in one or both sides of drug layer (J. Shokri et al., 2008b; Kumaravelrajan et al., 2010). These polymers should have high water uptake and swelling capacity. Cellulose derivatives play an important role in core formulations of osmotic devices. Water soluble cellulose ethers commonly used as core polymers due to their hydrophilicity and good swelling properties. Most currently used polymers for this purpose are MC, HEC, HPC and HPMC with various molecular weights. After exposing of system to water, water move into the system due to great osmotic pressure difference between outer and inner part of device. This water is imbibed to polymer(s) and causes swelling of them. Swelling of core polymer(s) produce the driving force for ejecting the drug solution from drug release orifice with constant rate (J. Shokri et al., 2008a, 2008b; Prabakaran et al., 2004; Makhija \& Vavia, 2003; Liu et al., 2000a, 2000b). Among cellulose ethers, different grades of HPMC have been used more than others in core formulation. Microcrystalline cellulose (MCC) has also frequently used the core formulations as compressibility enhancer. MCC is one of the most compressibility enhancers that widely used in direct compression as well as wet granulation techniques for preparing various types of tablets, pellets and pills.

\subsection{Application in enteric coated solid dosage form}

Enteric coated solid dosage forms are the main groups of delayed release drug delivery systems which designed for releasing of their $\operatorname{drug}(\mathrm{s})$ content in the lower parts of gastrointestinal tract such as small intestine and colon. Enteric dosage forms can be considered as a type of oral site specific pharmaceuticals that initiate drug release after passing from stomach. Enteric oral dosage forms are suitable for formulation of acid-labile drugs or drugs with irritancy potential for inner protective layer of stomach such as nonsteroidal anti inflammatory drugs (NSAIDs). The commonly used materials in enteric coated formulations are $\mathrm{pH}$-dependent polymers containing carboxylic acid groups. These polymers remain un-ionized in low $\mathrm{pH}$ conditions like environment of stomach and become ionized with increasing of $\mathrm{pH}$ toward natural and light alkaline zone similar to the small intestine condition (Liu et al., 2011). These polymers also should have the good film forming properties to produce smooth coats with good integrity. Various polymers have been used for production of enteric coated dosage forms such as Eudragit ${ }^{\circledR}$ polymers and $\mathrm{pH}$ dependent cellulose derivatives. Cellulose derivatives which commonly used as enteric 
coating polymers include cellulose acetate phthalate (CAP), cellulose acetate trimelitate (CAT), hydroxypropylmethyl cellulose phthalate (HPMCP), carboxymethylethyl cellulose (CMEC) and hydroxypropylmethyl cellulose acetate succinate (HPMCAP) (Williams III \& Liu, 2000). Apart from the main enteric polymer, the type and amount o plasticizer(s) is very important for achieving uniform, smooth and resistant enteric films. Some of mostly used plasticizers in enteric coated formulations are diethyl phthalate, glyceryl triacetate, glyceryl monocaprylate and triethyl citrate (Williams III \& Liu, 2000; Gosh et al., 2011). In some cases, hydrophilic cellulose ether derivatives especially HPMC are used with enteric polymer for improving the film forming and plasticity of main enteric polymer. HPMC is also used in enteric coating process as pre-coating or sub-coating polymer due to its very good film forming properties and suitable polymer-to-polymer adhesion with enteric coating polymers especially with cellulose ester derivatives such as CAP, HPMCP, HPMCAS, CMEC and CAT (Williams III \& Liu, 2000). Three commercially available enteric coating preparations included solid forms of enteric polymers which should be dissolved in suitable organic solvent mixture before coating process, ready-to-use organic enteric coating solutions and aqueous polymeric dispersions. Aqueous nanodispersions of enteric coating polymers such as HPMCP have also been investigated for improving physicochemical and mechanical characteristics of coating (Kim et al., 2003).

\subsection{Application as compressibility enhancers}

More than 80 percent of all dosage forms available or administered to man are tablets. The main reason of this great popularity is the advantages of tablets over other forms of pharmaceuticals. Ease of manufacturing, convenience dosing and greater stability in compared with liquid or semisolid dosage forms are some of these advantages. Two common ways for tablet manufacturing are compression and molding. Except of a few cases, tablets are made by compression technique. The simplest and fastest kind of compression is named direct compression method in which the drug and all of other excipients are mixed and compressed in one-stage process with proper compression force to form tablet. This method commonly used for tabletting of medium to high potency drugs where the drug content in them is less than $30 \%$ of formulation (Jivari et al., 2000). In the other cases with higher amounts of low compactable drugs, dry or wet granulation techniques are used for preparing tablets. In dry granulation method, compression of ingredients are performed in two or multi-stage process to improve compressibility of the ingredients. Slugging and roller compaction techniques used for initial compression of powder mixtures before final tabletting process.

One of the common difficulties in direct compression and dry granulation is low compactability of the drug content especially when the drug amount is higher than $30 \%$ of formulation. In these cases, an efficient compressibility enhancer can help to achieving a good tablet with pharmaceutically accepted characteristics. Although, all of the cellulose based polymers are good compactable, however special grades of microcrystalline cellulose exhibit excellent compatibility. These grades can significantly improve compressibility of low compactable powder mixtures so they are widely used as compressibility enhancers in 
tablet manufacturing by direct compression and dry granulation methods. Various grades of MCC have different fundamental properties including their morphology, particle size, surface area, porosity and density (Rojas \& Kumar, 2011). These physicochemical properties poses the different characteristics to them for example, smaller particle size MCC grades have good compressibility and poor flowability whereas the larger particle size grades have poor compressibility and excellent flowability. Particle size of MCC varies from 20 to 270 micrometer based on the manufacturer and type of application. MCC is available in three public brand names including Avicel $^{\circledR}$ (FMC BioPolymer), VIVAPUR ${ }^{\circledR} /$ EMCOCEL $^{\circledR}$ (JRS Pharma) and TABULOSE ${ }^{\circledR}$ (Blanver). Various grades of commercially available Avicel ${ }^{\circledR}$ and their particle size are shown in table 1 (Colorcon official website).

\begin{tabular}{|c|c|c|c|}
\hline brand name & Application & MCC grade & Particle size \\
\hline \multirow[t]{14}{*}{ Avicel $^{\circledR}$} & Roller compaction & DG & 45 \\
\hline & Wet granulation & PH-101 & 50 \\
\hline & \multirow{2}{*}{ Direct compression } & PH-102 & 100 \\
\hline & & HFE-102 & 100 \\
\hline & Superior compactability & PH-105 & 20 \\
\hline & \multirow[t]{2}{*}{ Superior Flowability } & PH-102 SCG & 150 \\
\hline & & PH-200 & 180 \\
\hline & \multirow{2}{*}{ High Density } & PH-301 & 50 \\
\hline & & PH-302 & 100 \\
\hline & \multirow[t]{4}{*}{ Low Humidity } & PH-103 & 50 \\
\hline & & PH-113 & 50 \\
\hline & & PH-112 & 100 \\
\hline & & PH-200LM & 180 \\
\hline & Mouthfeel improvement & CE-15 & 75 \\
\hline
\end{tabular}

Table 1. Various grenades of Avicel ${ }^{\circledR}$

The effects of size, shape and porosity of MCC particles on flowability and compatibility have also been investigated by several researchers (Johansson et al., 2001). Various types of MCCs are extensively used in direct compression and dry granulation methods especially in roller compaction for preparing compressed tablets or pellets (Strydom et al., 2011; Bultmann, 2002). Microcrystalline cellulose type II (MCC-II) was recently introduced as new pharmaceutical excipients. MCC-II has a fibrous structure with lower compactability than MCC grades and suitable for using in rapid disintegrating dosage forms (Rojas et al., 2011; Reus-Medina \& Kumar, 2006). In recent years, the new methods have been established for improving mechanical characteristics of MCCs. One of these innovative methods is lubricating or silisfying for improving compactability of low compressible grades of MCCs such as MCC-II or large particle size MCC grades. In this method, amorphous silicon dioxide $(\mathrm{SiO} 2)$ is used as companion excipient for co-processing with low compressible MCC grades. Cellulose/SiO2 ratio is $98: 2$ and resulted product is called lubricated or silisified microcrystalline cellulose (SMCC). This method can be used for both MCC-I or MCC-II for production SMCC-I or SMCC-II (Rojas \& Kumar, 2011; Van Veen et al., 2005). SMCC-I have excellent compaction properties and less stickiness to the lower punches 
over MCC-I or MCC-I/SiO2 physical mixtures (Rojas \& Kumar, 2011). SMCC-II has also better mechanical properties especially higher compactability than MCC-II without detriment if it's self-disintegrating characteristics. SMCC-I grades are commercially available under trade name of ProSolv ${ }^{\circledR}$ (JRS Pharma) but SMCC-II is not commercialized yet. Apart from MCC, other forms of cellulose are existed such as powdered cellulose (PC) and low cristallinity powdered cellulose (LCPC). LCPC and MCC have agglomerated and PC has fibrous structure. PC applications in pharmaceutical industries is similar that MCC. It is widely used in direct compression formulation and in dry granulation by either slugging or roller compaction methods. LCPC is a new direct compression cellulose excipient which is prepared by controlled decrystallization and depolymerization of cellulose with phosphoric acid (Rojas \& Kumar, 2011). LCPC was shown superior tabletting properties than direct compression grades of MCC like Avicel ${ }^{\circledR P H}-101$ (Kothari et al., 2002).

\subsection{Application as gelling agents}

Gels are semisolid systems consisting of dispersions of very small particles or large molecules in an aqueous liquid vehicle rendered jellylike by the addition of a gelling agent. In recent decades, synthetic and semi-synthetic macromolecules are mostly used as gelling agents in pharmaceutical dosage forms. Some of these agents include: carbomers, cellulose derivatives and natural gums. Cellulose derivatives such as HPMC and CMC are the most popular gelling agents used in drug formulations. These polymers are less sensitive for microbial contamination than natural gelling agents such as tragacanth, acacia, sodium algininate, agar, pectin and gelatin. Cellulose derivatives generally dissolve better in hot water (except MC grades) and their mechanisms of jellification is thermal. For preparing gel, powder of these polymers with suitable amount initially dispersed in cold water by using mechanical mixture and then, the dispersion is heated to about $60-80^{\circ} \mathrm{C}$ and gradually cooled to normal room temperature to form a gel (except MC grades). The resulted gels from these polymers are single-phase gels. Adding of electrolytes in the low concentrations increase the viscosity of these gels by salting out mechanism and higher concentrations (above 3-4\%) can precipitate the polymer and breakup the gel system (Allen, et al., 1995). Maximum stability and transparency of the gels prepared by these polymers is about neutral range $(\mathrm{pH}=7-9)$ and acidic $\mathrm{pHs}$ can precipitate them from gel system. Minimum gelforming concentrations of cellulose derivatives are different based on the type and the molecular weights of them but the medium range is about $4-6 \% \mathrm{w} / \mathrm{v}$. The type of cellulose derivative in pharmaceutical gels can significantly affect drug release from gel formulations (Tas, et al., 2003). These gels also can be used as the base of novel drug delivery systems such as liposomal formulations (Gupta, et al., 2012).

\subsection{Application as thickening and stabilizing agents}

Cellulose derivatives are extensively used for thickening of pharmaceutical solutions and disperse systems such as emulsions and suspensions (Adibkia et al., 2007a, 2007b). Furthermore, these polymers can increase viscosity of non-aqueous pharmaceutical solution likes organic-based coating solutions. Viscosity enhancing of drug solutions poses many 
advantages such as improving consuming controllability and increasing residence time of drugs in topical and mucosal solutions which lead to improve bioavailability of topical, nasal or ocular preparations (Grove et al., 1990; Adibkia et al., 2007a, 2007b). It has been revealed that viscosity enhancement, in some cases, can increase absorption of some poorlyabsorb drugs like insulin from oral dosage forms (Mesiha, M. \& Sidhom, M.). Cellulose ethers in concentrations lower than minimum gel-forming amounts are used as thickening agents or viscosity builder. These polymers play an important role in stabilizing of pharmaceutical disperse systems especially in suspensions and coarse emulsions. By increasing the viscosity of suspension, based on the stock's equation, the sedimentation rate of dispersant decreased and thus, the uniformity of dispersion after shaking of product will improve. In the case of emulsions, these polymers can increase the shelf life and their resistance against mechanical and thermal shocks. Among cellulose derivatives, cellulose ethers especially their higher molecular weight grades are more suitable for using as viscosity enhancer and stabilizer for liquid pharmaceutical disperse systems such as suspensions and emulsions. There is a direct proportionality between viscosity of cellulose ether solutions and molecular weights of them.

\subsection{Application as fillers in solid dosage forms}

Cellulose and related polymers are commonly used in solid dosage forms like tablets and capsules as filler. Various forms of cellulose have been used in pharmaceutical preparations as multifunctional ingredients thus; they are concerned as precious excipients for formulation of solid dosage forms. Cellulose and its derivatives have many advantages in using as filler in solid pharmaceuticals such as their compatibility with the most of other excipients, pharmacologically inert nature and indigestibility by human gastrointestinal enzymes. These polymers do not cause any irritancy potential on stomach and esophagus protective mucosa. Various forms of pure cellulose and cellulose ether derivatives can be used as filler in these formulations.

\subsection{Application as binders in granulation process}

Binders are the essential components of solid drug formulations made by wet granulation process. In wet granulation process, drug substance is combined with other excipients and processed with the use of a solvent (aqueous or organic) with subsequent drying and milling to produce granules. Cellulose and some derivatives have excellent binding effects in wet granulation process. A number of MCC grades such as $\mathrm{PH}-101$ are widely used as binder in wet granulation. Other cellulose derivatives such as MC, HPMC and HPC have good binding properties in wet granulation. Low substituted cellulose ethers such as low substituted HPC (L-HPC) also used as binder in wet granulation process (Desai et al., 2006; Wan \& Prasad, 1988). Even though, low substituted cellulose ethers have lower water solubility compared with normal grades, however they have very good binding efficacy. Cross-linked cellulose (CLC) and cross-linked cellulose derivatives such as cross-linked $\mathrm{NaCMC}$ can be used as excellent binders in pharmaceuticals as well (Chebli \& Cartilier, 1998). 


\subsection{Application as disintegrating agents}

Solid oral dosage forms such as tablets undergo several steps before systemic absorption of the drug. Disintegration is the first step immediately after administration of oral dosage forms that breakup the dosage forms into the smaller fragments in an aqueous environment. Converting of solid dosage forms to smaller fragments, increase the available surface area and promote a more rapid release of the drug substances from dosage forms. The earliest known disintegrant is Starch. Corn Starch or Potato Starch was recognized as being the ingredient in tablet formulations responsible for disintegration as early as 1906. Due to low compressibility of starch, pre-gelatinized starch was invented for using as disintegrant. Pregelatinized starch and MCC are two main types of classic disintegrants. In recent years, the classic disintegrnts have been gradually replaced with the newer ones called super disintegrants. Super disintegrants can acts in lower concentrations than starch and have not detriment effect on compressibility and flowability of formulations. Three main groups of these excipients are: modified starches like sodium starch glycolate (Primogel $\AA$, Explotab $\AA$ ) with 4-6\% effective concentration, cross-linked polyvinyl pyrrolidones like crospovidone (Polyplasdone XL, Kollidon CL) with 2-4\% effective concentration and modifies cellulose like cross-linked sodium carboxymethyl cellulose or croscarmellose (Ac-Di-Sol ${ }^{\mathrm{TM}}$ and Nymcel) with 2-4\% effective concentration in wet granulation process. Modified cellulose compounds are very efficient disintegrants and additionally, can accelerate the dissolution rate of drugs in aqueous environment (Chebli \& Cartilier, 1998).

\subsection{Application as taste masking agents}

There are numerous drugs with unfavorable tastes. The most prevalent unpleasant taste of the drugs is bitter taste. Unpleasant-tasting dosage forms leads to lack of patient compliance of oral drug preparations. Various tastes are feeling by taste buds on the tongue. Taste buds are onion-shaped structures containing between 50 to 100 taste cells. Chemicals from food or oral ingested medicine are dissolved by the saliva and enter via the taste pore. They either interact with surface proteins known as taste receptors or with pore-like proteins called ion channels. These interactions cause electrical changes within the taste cells that trigger them to send chemical signals that translate into neurotransmission to the brain. Salt and sour responses are of the ion channel type of responses, while sweet and bitter are surface protein responses.

Taste masking is an important consideration in formulation of oral dosage forms especially in the case of high dose, poorly tasting drugs. Improving the taste of liquid dosage forms is more important because of better sensitivity and faster stimulation of taste receptors by liquids in compared than solids. Taste masking in solid dosage forms can be performed by coating (in the case of tablets, pellets, pills or coarse granules) or micro-coating (in the case of fine granules, powders or microcapsules) of them by a gastro-soluble polymeric coating. These coats can prevent from contacting of the drug with taste buds without detriment of release characteristics of the drug formulations in gastrointestinal tract. Soluble cellulose ether derivatives are suitable for this purpose. These polymers like HPMC, HEC, MC and 
HPC are completely water soluble and they have very good film forming properties. Some grades of MCC also can improve tooth-feel such as Avicel ${ }^{\circledR}$ CE-15. These coats can produce additional benefits in drug formulations such as protection of the active ingredients against moisture, oxygen of the air and light due to their barrier effects. Masking of the taste in liquid dosage forms especially in drug solutions is more sophisticated. In these cases test receptor blockers, flavoring agents and viscosity enhancers are simultaneously needed.

\section{Author details}

Javad Shokri and Khosro Adibkia

Faculty of Pharmacy, Tabriz University of Medical Sciences, Tabriz, Iran

\section{References}

Adibkia, k.; Hamedeyazdan, S.; Javadzadeh, Y. (2011). Drug release kinetics and physicochemical characteristics of floating drug delivery systems. Expert Opinion on Drug Delivery, Volume 8, Issue 7 (July), Pages 891-903.

Adibkia, K.; Omidi, Y.; Siahi Shadbad, MR.; Nokhodchi, A.; Javadzedeh, A.; Barzegar-Jalali, M.; Barar, J.; Mohammadi, G. (2007b). Inhibition of endotoxin-induced uveitis by methylprednisolone acetate nanosuspension in rabbits. Journal of Ocular Pharmacology and Therapeutics, Volume 23, Issue 5 (May), Pages 421-432.

Adibkia, K.; Siahi Shadbad, MR.; Nokhodchi, A.; Javadzedeh, A.; Barzegar-Jalali, M.; Barar, J.; Mohammadi, G.; Omidi, Y. (2007a). Piroxicam nanoparticles for ocular delivery: Physicochemical characterization and implementation in endotoxin-induced uveitis. Journal of Drug Targeting, Volume 15, Issue 6 (June), Pages 407-416.

Allen, L.V.; Popovich, N.G.; Ansel, H.C. (1995). Ansels Pharmaceutical Dosage Forms and Drug Delivery ayatems (8 $8^{\text {th }}$ Edition), Lippincott Williams \& Wilkins, ISBN: 0-7817-46124, United States of America

Arwidsson, H. \& Nicklasson, M. (1990). Application of intrinsic viscosity and interaction constant as a formulation tool for film coating II. Studies on different grades of ethyl cellulose in organic solvent systems. International Journal of Pharmaceutics, Volume 58, Issue 1 (15 January), Pages 73-77.

Asnaashari, S.; Khoei, NS.; Zarrintan, MH.; Adibkia, K.; Javadzadeh, Y. (2011). Preparation and evaluation of novel metronidazole sustained release and floating matrix tablets. Pharmaceutical Development and Technology, Volume 16, Issue 4 (April), Pages 400-407.

Barzegar-Jalali, M.; Valizadeha, H.; Dastmalchi, S.; Siahi Shadbad, MR.; Barzegar-Jalal, A.; Adibkia, K.; Mohammadi, G. (2007). Enhancing dissolution rate of carbamazepine via cogrinding with crospovidone and hydroxy propyl methyl cellulose. Iranian Journal of Pharmaceutical Research, Volume 6, Issue 3 (March), Pages 159-165.

Barzegar-Jalali, M.; Valizadeha, H.; Siahi Shadbad, MR.; Adibkia, K.; Mohammadi, G.; Farahani, A.; Arash, Z.; Nokhodchi, A. (2010). Cogrinding as an approach to enhance dissolution rate of a poorly water-soluble drug (gliclazide). Powder Technology, Volume 197, Issue 3 (March), Pages 150-158. 
Bultmann, J.M. (2002). Multiple compaction of microcrystalline cellulose in a roller compactor. European Journal of Pharmaceutics and Biopharmaceutics, Volume 54, pages 5964.

Chebli, C. \& Cartilier, R. (1998). Cross-linked cellulose as a tablet excipient: A binding/disintegrating agent. International Journal of Pharmaceutics, Volume 171, Pages 101-110.

Chopra, Sh.; Patil, G.V.; Motwani, S.K. (2007). Release modulating hydrophilic matrix systems of losartan potassium: Optimization of formulation using statistical experimental design. European Journal of Pharmaceutics and Biopharmaceutics, Volume 66, Issue 1 (April), Pages 73-82.

Colorcon

official

website, http//fmcbiopolymer.com/pharmaceutical/product/Avicelforsoliddosageforms/ASPX

Conti, S.; Maggi, L.; Segale, L.; Machiste, E.O.; Conte, U.; Grenier, P.; Vergnault, G. (2007). Matrices containing NaCMC and HPMC: 1. Dissolution performance characterization. International Journal of Pharmaceutics, Volume 333, Issues 1-2 (21 March), Pages 136-142.

Desai, D.; Rinaldi,F.; Kothari, S.; Paruchuri, S.; Li, M.; Lai, D.; Fung, S.; Both, D. (2006). Effect of hydroxypropyl cellulose (HPC) on dissolution rate of hydrochlorothiazide tablets International Journal of Pharmaceutics, Volume 308, Pages 40-45.

Deshpande, M.C.; Venkateswarlu, V.; Babu, R.K.; Trivedi, R.K. (2009). Design and evaluation of oral bioadhesive controlled release formulations of miglitol, intended for prolonged inhibition of intestinal $\alpha$-glucosidases and enhancement of plasma glucagon like peptide-1 levels. International Journal of Pharmaceutics, Volume 380, Issues 1-2, (1 October), Pages 16-24.

Ferrero, C.; Massuelle, D.; Doelker, E. (2010). Towards elucidation of the drug release mechanism from compressed hydrophilic matrices made of cellulose ethers. II. Evaluation of a possible swelling-controlled drug release mechanism using dimensionless analysis. Journal of Controlled Release, Volume 141, Issue 2 (25 January), Pages 223-233.

Ferrero, C.; Massuelle, D.; Jeannerat, D.; Doelker, E. (2008). Towards elucidation of the drug release mechanism from compressed hydrophilic matrices made of cellulose ethers. I. Pulse-field-gradient spin-echo NMR study of sodium salicylate diffusivity in swollen hydrogels with respect to polymer matrix physical structure. Journal of Controlled Release, Volume 128, Issue 1 (22 May), Pages 71-79.

Frohoff-Hülsmann, M.A.; Lippold, B.C.; McGinity, J.W. (1999a). Aqueous ethyl cellulose dispersion containing plasticizers of different water solubility and hydroxypropyl methyl- cellulose as coating material for diffusion pellets II: properties of sprayed films. European Journal of Pharmaceutics and Biopharmaceutics, Volume 48, Issue 1 (1 July), Pages 67-75.

Frohoff-Hülsmann, M.A.; Schmitz, A.; Lippold, B.C. (1999b). Aqueous ethyl cellulose dispersions containing plasticizers of different water solubility and hydroxypropyl methylcellulose as coating material for diffusion pellets: I. Drug release rates from coated pellets. International Journal of Pharmaceutics, Volume 177, Issue 1 (15 January), Pages 69-82. 
Ghosh, I.; Snyder, J.; Vippagunta, R.; Alvine, A.; Vakil, R.; Tong W-Q,; Vippagunta, S. (2011). Comparison of HPMC based polymers performance as carriers for manufacture of solid dispersions using the melt extruder. International Journal of Pharmaceutics, Volume 419, Issues 1-2, (31 October), Pages 12-19.

Grabovac, V.; Guggi, D.; Bernkop-Schnurch, A. (2005). Comparison of the mucoadhesive properties of various polymers. Advanced Drug Delivery Reviews. Volume 57, Pages 1713- 1723.

Grove, J.; Durr, M.; Quint, M-P.; Plazonnet. B. (1990). The effect of vehicle viscosity on the ocular bioavailability of L-653,328. International Journal of Pharmaceutics, Volume 66, Issues 1-3, ( December 1990), Pages 23-28

Gupta, P.N.; Pattani, A.; Curran, R.M.; Kett, V.L.; Andrews, G.P.; Morrow, R.J.; Woolfson, A.D.; Malcolm, R.K. (2012). Development of liposome gel based formulations for ntravaginal delivery of the recombinant HIV-1 envelope protein CN54gp140, European Journal of Pharmaceutical Sciences, In Press, Corrected Proof, Available online 14 February 2012.

Heinämäki, J.T.; Iraizoz Colarte, A.; Nordström, A.J.; Yliruusi, J.K. (1994). Comparative evaluation of ammoniated aqueous and organic-solvent-based celluloseester enteric coating systems: a study on free films. International Journal of Pharmaceutics, Volume 109, Issue 1 (22 August), Pages 9-16.

Hjärtstam, J.; Borg, K.; Lindstedt, B. (1990). The effect of tensile stress on permeability of free films of ethyl cellulose containing hydroxypropyl methylcellulose. International Journal of Pharmaceutics, Volume 61, Issues 1-2 (11 June), Pages 101-107.

Javadzadeh, Y.; Hamedeyazdan, S.; Adibkia, K.; Kiafar, F.; Zarrintan, MH.; Barzegar-Jalali, M. (2010). Evaluation of drug release kinetics and physicochemical characteristics of metronidazole floating beads based on calcium silicate and gas forming agents. Pharmaceutical Development and Technology, Volume 15, Issue 4 (April), Pages 329-338.

Jivraj, M.; Martini, L.G.; Thomson, C.M. (2000). An overview of the different excipients useful for the direct compression of tablets. Pharmaceutical Science $\mathcal{E}$ Technology Today, Volume 3, Issue 2, (1 February), Pages 58-63.

Johansson, B. \& Alderborn, G. (2001). The effect of shape and porosity on the compression behavior and tablet forming ability of granular materials formed from microcrystalline cellulose, European Journal of Pharmaceutics and Biopharmaceutics, Volume 52, Issue 3 (November), Pages 347-357.

Kangarlou, S.; Haririan, I.; Gholipour, Y. (2008). Physico-mechanical analysis of free ethyl cellulose films comprised with novelplasticizers of vitamin resources. International Journal of Pharmaceutics, Volume 356, Issues 1-2 (22 May), Pages 153-166.

Karasulu, Y.H.; Hilmioğlu, S.; Metin, D.Y.; Güneri T. (2004). Efficacy of a new ketoconazole bioadhesive vaginal tablet on Candida albicans.Il Farmaco, Volume 59, Issue 2 (February), Pages 163-167.

Karavas, E.; Georgarakis, E.; Bikiaris, D. (2006). Application of PVP/HPMC miscible blends with enhanced mucoadhesive properties for adjusting drug release in predictable pulsatile chronotherapeutics. European Journal of Pharmaceutics and Biopharmaceutics, Volume 64, Issue 1 (August), Pages 115-126. 
Kim, H.; Park, J.H.; Cheong, I.W.; Kim, J.H. (2003). Swelling and drug release behavior of tablets coated with aqueous hydroxypropyl methylcellulose phthalate (HPMCP) nanoparticles. Journal of Controlled Release, Volume 89, Issue 2 (29 April), Pages 225-233.

Kothari, S.H.; Kumar, V.; Banker, G.S. (2002). Comparative evaluations of powder and mechanical properties of low crystallinity celluloses, microcrystalline celluloses, and powdered celluloses. International Journal of Pharmaceutics, Volume 232, Issues 1-2 (31 January), Pages 69-80.

Kumaravelrajan, R.; Narayanan, N.; Suba, V.; Bhaskar, K. (2010). Simultaneous delivery of Nifedipine and Metoprolol tartarate using sandwiched osmoticpump tablet system. International Journal of Pharmaceutics, Volume 399, Issues 1-2 (31 October), Pages 60-70.

Larsson, M.; Hjärtstam, J.; Berndtsson, J.; Stading, M.; Larsson, A. (2010). Effect of ethanol on the water permeability of controlled release films composed of ethyl cellulose and hydroxypropyl cellulose. European Journal of Pharmaceutics and Biopharmaceutics, Volume 76, Issue 3 (November), Pages 428-432.

Lecomte, F.; Siepmann, J.; Walther, M.; MacRae, R.J.; Bodmeier, R. (2003). Blends of enteric and GIT-insoluble polymers used for film coating: physicochemical characterization and drug release patterns. Journal of Controlled Release, Volume 89, Issue 3 (20 May), Pages 457-471.

Lehr, Claus-Michael. (2000). Lectin-mediated drug delivery: The second generation of bioadhesives Journal of Controlled Release. 65, Pages 19-29.

Liu, F.; Merchant, H.A.; Kulkarni, R.P.; Alkademi, M.; Basit, A.W. (2011). Evolution of a physiological $\mathrm{pH} 6.8$ bicarbonate buffer system: Application to the dissolution testing of enteric coated products. European Journal of Pharmaceutics and Biopharmaceutics, Volume 78, Issue 1 (May), Pages 151-157.

Liu, J. \& Williams, R.O. (2002). Long-term stability of heat-humidity cured cellulose acetate phthalate coatedbeads. European Journal of Pharmaceut cs and Biopharmaceutics, Volume 53, Issue 2 (March), Pages 167-173.

Liu, L.; Khang, G.; Rhee, J.M.; Lee, H.B. (2000a). Monolithic osmotic tablet system for nifedipine delivery. Journal of Controlled Release, Volume 67, Issues 2-3 (3 July), Pages 309322.

Liu, L.; Ku, J.; Khang, G.; Lee, B.; Rhee, J.M.; Lee, H.B. (2000b). Nifedipine controlled delivery by sandwiched osmotic tabletsystem. Journal of Controlled Release, Volume 68, Issue 2 (10 August), Pages 145-156.

Ludwig, Annick.; (2005). The use of mucoadhesive polymers in ocular drug delivery. Advanced Drug Delivery Reviews. Volume 57, Pages 1595- 1639.

Makhija S.N. \& Vavia, P.R. (2003). Controlled porosity osmotic pump-based controlled release systems ofpseudoephedrine: I. Cellulose acetate as a semipermeable membrane. Journal of Controlled Release, Volume 89, Issue 1 (14 Apri), Pages 5-18.

Marucci, M.; Ragnarsson, G.; Nilsson, B.; Axelsson, A. (2010). Osmotic pumping release from ethyl-hydroxypropyl-cellulose -coated pellets: A new mechanistic model. Journal of Controlled Release, Volume 142, Issue 1 (25 February), Pages 53-60. 
Mesiha, M.; Sidhom, M. (1995). Increased oral absorption enhancement of insulin by medium viscosity hydroxypropyl cellulose. International Journal of Pharmaceutics, Volume 114, Issue 2, (14 February), Pages 137-140.

Movassaghian, S.; Barzegar-Jalali, M.; Alaeddini, M.; Hamedyazdan, S.; Afzalifar, R.; ZakeriMilani, P.; Mohammadi, G.; Adibkia K. (2011). Development of amitriptyline buccoadhesive tablets in management of pain in dental procedures. Drug Development and Industrial Pharmacy, Volume 37, Issue 7 (June), Pages 1-12.

Nerurkar, J.; Jun, H.W.; Price, J.C.; Park, M.O. (2005). Controlled-release matrix tablets of ibuprofen using cellulose ethers and carrageenans: effect of formulation factors on dissolution rates. European Journal of Pharmaceutics and Biopharmaceutics, Volume 61, Issues 1-2 (September), Pages 56-68.

Nokhodchi, A.; Momin, M.N.; Shokri, J.; Shahsavari, M.; Rashidi, P.A. (2008). Factors Affecting the Release of Nifedipine from a Swellable Elementary Osmotic Pump. Drug Delivery, volume 15, Pages 43-48.

Okimoto, K.; Ohike, A.; Ibuki, R.; Aoki, O.; Ohnishi, N.; Rajewski, R.A.; Stella, V.J.; Irie, T.; Uekama, K. (1999). Factors affecting membrane-controlled drug release for an osmotic pump tablet (OPT) utilizing (SBE) $7 \mathrm{~m}-\beta-\mathrm{CD}$ as both a solubilizer and osmotic agent. Journal of Controlled Release, Volume 60, Issues 2-3 (5 August), Pages 311-319.

Perioli, L.; Ambrogi, V.; Rubini, D.; Giovagnoli, S.; Ricci, M.; Blasi, P.; Rossi, C. (2004). Novel mucoadhesive buccal formulation containing metronidazole for the treatment of periodontal disease. Journal of Controlled Release, Volume 95, Issue 3, 24 (March), Pages 521-533.

Prabakaran, D.; Singh, P.; Kanaujia, P.; Jaganathan, K.S.; Rawat, A.; Vyas, S.P. (2004). Modified push-pull osmotic system for simultaneous delivery of theophylline andsalbutamol: development and in vitro characterization. International Journal of Pharmaceutics, Volume 284, Issues 1-2, (13 October), Pages 95-108.

Pygall, S.R.; Kujawinski, S.; Timmins, P.; Melia, C.D. (2009). Mechanisms of drug release in citrate buffered HPMC matrices. International Journal of Pharmaceutics, Volume 370, Issues 1-2 (31 March), Pages 110-120.

Reus Medina, M.L. \& Kumar, V. (2006). Evaluation of cellulose II powders as a potential multifunctional excipient in tablet formulations. International Journal of Pharmaceutics, Volume 322, Pages 31-35.

Rojas, J. \& Kumar, V. (2011). Comparative evaluation of silicified microcrystalline cellulose II as a direct compression vehicle. International Journal of Pharmaceutics, Volume 416, Issue 1 (15 September), Pages 120-128.

Rowe, R.C. (1986). The effect of the molecular weight of ethyl cellulose on the drug release properties ofmixed films of ethyl cellulose andhydroxypropylmethylcellulose. International Journal of Pharmaceutics, Volume 29, Issue 1 (March), Pages 37-41.

Roy, D.S. \& Rohera, B.D. (2002). Comparative evaluation of rate of hydration and matrix erosion of HEC and HPC and study of drug release from their matrices. European Journal of Pharmaceutical Sciences, Volume 16, Issue 3, August 2002, Pages 193-199. 
Saša, B.; Odon, P.; Stane, S.; Julijana, K. (2006). Analysis of surface properties of cellulose ethers and drug release from their matrix tablets. European Journal of Pharmaceutical Sciences, Volume 27, Issue 4 (March), Pages 375-383.

Sensoy, D.; Cevher, H.; Sarıcı, A.; Yılmaz, M.; Özdamar, A.; Bergişadi, N. (2009). Bioadhesive sulfacetamide sodium microspheres: Evaluation of their effectiveness inthe treatment of bacterial keratitis caused by Staphylococcus aureus and Pseudomonasaeruginosa in a rabbit model. European Journal of Pharmaceutics and Biopharmaceutics, Volume 72, Issue 3 (August), Pages 487-495.

Shokri, J.; Ahmadi, P.; Rashidi, P.; Shahsavari, M.; Rajabi-Siahboomi, A.; Nokhodchi A. (2008a). Swellable elementary osmotic pump (SEOP): An effective device for delivery of poorly water-soluble drugs. European Journal of Pharmaceutics and Biopharmaceutics, volume 68, Pages 289-297.

Shokri, J.; Alizadeh, M.; Hassanzadeh, D.; Motavalli, F. (2008b). Evaluation of various parameters on release of indomethacin from two-layered core osmotic pump. Pharmaceutical Sciences (Tabriz Faculty of Pharmacy Journal), Volume 3 (winter), Pages 1322.

Shokri, M.H.; Arami, Z.; Shokri, J. (2011). Evaluation of formulation related parameters on the release of gliclazide from controlled porosity osmotic pump system. Pharmaceutical Sciences (Tabriz Faculty of Pharmacy Journal), 2011, Volume 16 (4), Pages 249-260.

Strydom, S.J.; Otto, D.P.; Liebenberg, W.; Lvov, Y.M.; Villiers, M.M. (2011). Preparation and characterization of directly compactible layer-by-layer nanocoated cellulose. International Journal of Pharmaceutics, Volume 404, Issues 1-2 (14 February), Pages 57-65.

Tas, Ç.; Özkan, Y.; Savaser, A.; Baykara, T. (2003). In vitro release studies of chlorpheniramine maleate from gels prepared by different cellulose derivatives. Il Farmaco, Volume 58, Issue 8 (August), Pages 605-611.

Van Veen, B.; Bolhuis, G.K.; Wu, Y.S.; Zuurman, K.; Frijlink, H.W. (2005). Compaction mechanism and tablet strength of unlubricated and lubricated (silicified) microcrystalline cellulose, European Journal of Pharmaceutics and Biopharmaceutics, Volume 59, Issue 1 (January), Pages 133-138.

Venkatesan, N.; Yoshimitsu, J.; Ohashi, Y.; Ito, Y.; Sugioka, N.; Shibata, N.; Takada, K. (2006) Pharmacokinetic and pharmacodynamic studies following oral administration of erythropoietin mucoadhesive tablets to beagle dogs. International Journal of Pharmaceutics, Volume 310, Issues 1-2, 9 (March), Pages 46-52.

Wan, L.S.C. \& Prasad, K.P.P. (1988). Effect of microcrystalline cellulose and cross-linked sodium carboxymethylcellulose on the properties of tablets with methylcellulose as a binder, Internafronal Journal of Pharmaceutics, Volume 41, Pages 159-167.

Wang, C.Y.; Ho, H-O.; Lin, L-H.; Lin, Y-K.; Sheu, M-T. (2005). Asymmetric membrane capsules for delivery of poorly water-soluble drugs byosmotic effects. International Journal of Pharmaceutics, Volume 297, Issues 1-2 (13 June), Pages 89-97.

Williams III, R.O \& Liu, J. (2000). Influence of processing and curing conditions on beads coated with an aqueous dispersion of cellulose acetate phthalate. European Journal of Pharmaceutics and Biopharmaceutics, Volume 49, Pages 243-252. 